\title{
Size-dependent K-edge EXAFS study of the structure of free Ar clusters
}

\author{
S. Kakar, ${ }^{*}$ O. Björneholm,${ }^{\dagger}$ J. Weigelt, A. R. B. de Castro, ${ }^{\ddagger}$ L. Tröger, R. Frahm, ${ }^{\S}$ and T. Möller \\ Hamburger Synchrotronstrahlungslabor HASYLAB at Deutsches Elektronen-Synchrotron DESY, Notkestrasse 85, \\ D-22603 Hamburg, Germany \\ A. Knop and E. Rühl**
Institut für Physikalische und Theoretische Chemie, Freie Universität Berlin, Takustrasse 3, D-14195 Berlin, Germany
}

(Received 3 September 1996)

\begin{abstract}
Extended x-ray absorption fine structure (EXAFS) spectroscopy is used to study the size-dependent structural evolution of free clusters. $K$-edge EXAFS data are obtained for Ar clusters in beams having mean cluster size ranging from 12 to 2900 atoms per cluster. EXAFS analysis yields size-dependent features which indicate the presence of an fcc structure at $\langle\mathrm{N}\rangle \geq 200$. It has been proposed in the literature that small rare-gas clusters have an icosahedral structure. The transition of this structure to the experimentally observed fcc lattice of rare-gas solids has been of long-standing interest and debate. The implications of our results are discussed in this light. [S0031-9007(97)02525-8]
\end{abstract}

PACS numbers: $36.40 . \mathrm{Mr}, 61.10 . \mathrm{Ht}$

Extended x-ray absorption fine structure (EXAFS) spectroscopy has been a valuable probe of local structure in molecules, surfaces, and solids [1]. It is to be expected that this technique is also useful for the study of the cluster structure and its evolution with cluster size, a problem which is of central interest to cluster physics. This Letter presents the results of the first size-dependent EXAFS study of free clusters and highlights the role that EXAFS spectroscopy can play in discerning the evolution of their structure. In this model study of $\mathrm{Ar}$ clusters $K$-edge EXAFS data are obtained for clusters of various sizes in order to study the long-standing problem of the structure of rare-gas clusters. Though there has been considerable theoretical effort in this direction, several unanswered questions regarding the structure of these clusters persist. In particular, it has been postulated that small rare-gas clusters have a noncrystalline polyicosahedral and multishell icosahedral structure (MIC) [2], and the nature of their transition to the crystalline fcc geometry is a matter of an ongoing debate [2-5]. Numerous theoretical simulations performed to estimate the cluster size for which the icosahedral structure is energetically preferable over an fcc cuboctahedral have yielded estimates that range from 1500 to $10^{4}$ atoms [3]. A great number of these theoretical efforts have strived to explain the results of the only direct experimental study available to date on this issue [2]. This study investigated the electron diffraction patterns for free Ar clusters, and concluded that the transition from icosahedral to fcc structure was discernible in cluster beams having clusters containing around 750 atoms. However, repeated theoretical efforts to simulate these experimental data, even with imperfect fcc crystals and for sizes for which the features characteristic of an fcc structure are visible, have failed. To overcome this problem the plausibility of the cluster growth process has been cited as a mechanism determining the cluster structure [4,5]. Based on this premise, a recent study suggested that larger clusters consist of a noncrystalline core of fivefold symmetry embedded in fcc crystalline material and that there is no size-dependent structural transition [5]. It is the goal of the present study to provide alternative experimental data to help resolve these issues and guide theory towards a consensus. By probing structural imprints in the EXAFS the study aims to discern the link between the cluster size and cluster structure, and whether there is a transition of structures during the cluster growth process.

While the preceding discussion presents the scientific case for the present work, it is also important to note its experimental significance. Though EXAFS spectroscopy is a powerful structural tool in materials science, its application to the study of free clusters is still in infancy. Clearly, the greatest benefits of this technique would be realized if data are obtained for clusters of various sizes and the size-dependent features in these data are isolated accurately. Unfortunately, this is a formidable challenge owing to the dilute nature of cluster beams. Early attempts in this direction produced data of poor statistics and covered, at best, a narrow size-range [6]. This rendered the determination of size-dependent effects difficult and yielded information limited in structural content. However, recent experimental advances, such as the development of brighter synchrotron radiation sources and cluster-specific detection methods [7], have made it possible in this study to obtain size-dependent high quality EXAFS data for free clusters. Moreover, it is determined that these data can indeed provide useful structural information, which holds promise for a new range of experiments involving EXAFS investigations of free clusters.

Experimental details are described elsewhere [7], and only a brief description is given here. An intense beam of Ar clusters is produced by an adiabatic expansion through a nozzle. The size distribution in the cluster 
beam is characterized by a mean cluster size $\langle N\rangle$ and width $\Delta N \approx\langle N\rangle$ [8]. $\langle N\rangle$ is estimated from empirical scaling laws and easily altered by changing the stagnation conditions [8]. There are alternative standards available for calculating $\langle N\rangle$ which give slightly different results [8]. In general, the discrepancy between these standards is less than $10 \%$ for cluster sizes $\leq 200$, and increases to about $50 \%$ for the largest clusters considered here. The cluster beam is ionized by synchrotron radiation from the undulator beam line BW1 at HASYLAB, Hamburg. This beam line provides a high photon flux and utilizes a mirror bender for matching the x-ray beam size with the sample size, thereby making this experiment with dilute cluster beams possible.

The energy of the synchrotron radiation is tuned to excite the Ar $1 s$ electron. The core excitation of a cluster atom leads to an efficient fragmentation of the cluster and, except for the very large parent clusters, the subsequent ion yield consists primarily of singly charged monomer and dimer ions [9]. We are thus able to monitor the x-ray absorption of clusters by measuring the partial ion yield of these ions by a time-of-flight mass spectrometer. Note that these measurements are cluster specific and enable us to observe weak cluster effects even when the degree of condensation in the cluster beam is low [7].

Figure 1 shows the experimental $\mathrm{x}$-ray absorption $\mu$ of Ar clusters of mean size $\langle N\rangle$ ranging from 12 to 2900 atoms. The energy range covers the Ar $K$-edge and extends to about $180 \mathrm{eV}$ above the edge. The spectrum for the largest clusters bears a close resemblance to that for the solid $\mathrm{Ar}$ in [10], indicating that the important size range has been sampled. The spectra show a prominent peak at around $3203.5 \mathrm{eV}$ due to the $1 s \rightarrow 4 p$ transition, and this is followed by features which are absent in the atomic spectrum. This fine structure in the absorption originates from the scattering of the outgoing photoelectrons by the surrounding atoms and the subsequent interference of the scattered and the parent waves [1]. In this work we are interested in the EXAFS which manifests itself as weak sinusoidal undulations beyond $3225 \mathrm{eV}$ in above spectra and has embedded in it the information regarding the mean local geometry [1].

The data presented in Fig. 1 are subjected to conventional EXAFS analysis to extract the structural information [1] using the UWEXAFS software [12]. The details of this analysis will be given in a subsequent paper. Briefly, we first determine the normalized magnitude of EXAFS $\chi$ as a function of the photoelectron momentum $k$. The result is then $k^{2}$ weighted and Fourier transformed to the $r$ space, where $r$ is the distance from the absorbing atom. Apart from corrections to $r$ values arising from the scattering phase shifts [1], the amplitude of the Fourier transform $\left|\mathrm{FT}\left[k^{2} \chi(k)\right]\right|$ reflects the mean local radial distribution function (MLRDF) for the cluster with peaks corresponding to various coordination shells of the absorbing atom: the area under the $j$ th peak depends on the

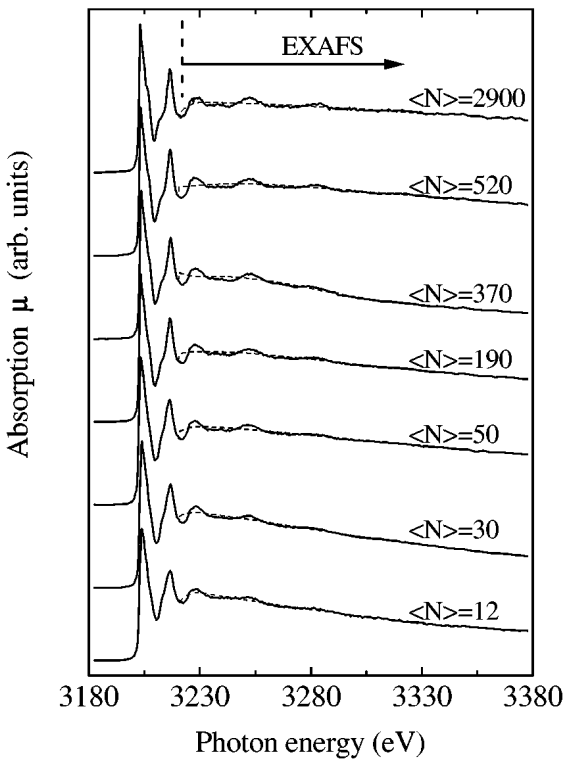

FIG. 1. X-ray absorption $\mu$ of Ar clusters at the Ar $K$-edge. $\langle N\rangle$ is the mean cluster size in the molecular beam.

mean value of the $j$ th coordination number [1]. These MLRDFs for various cluster sizes are shown in the Fig. 2.

To glean insights from the experimental data, the FEFF software package [13] is used to perform theoretical simulations for clusters having fcc and icosahedral structures. The cluster size is varied to decipher

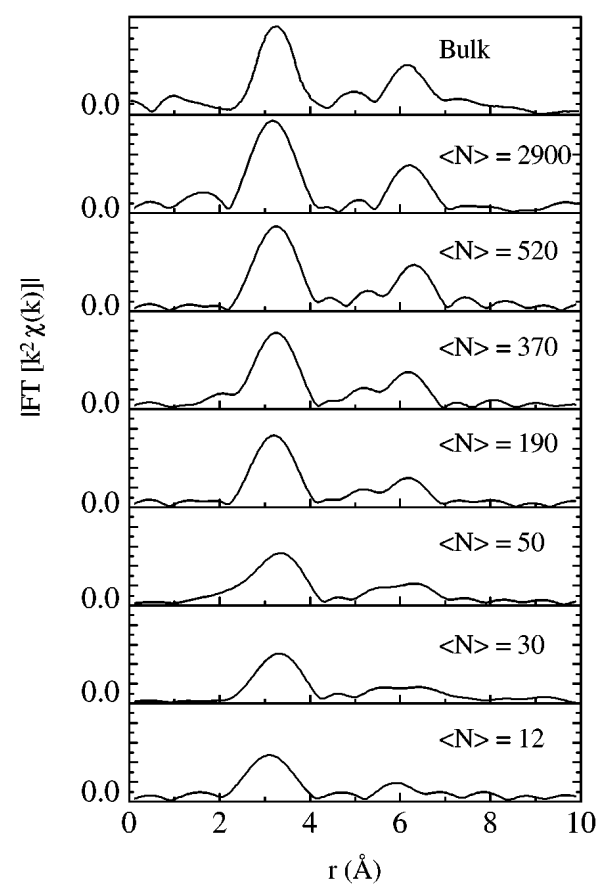

FIG. 2. Experimentally determined dependence of the mean radial distribution function $\left|\mathrm{FT}\left[k^{2} \chi(k)\right]\right|$ on $\langle N\rangle(2.1 \leq k \leq$ 5.9, Hanning window sills, $d k=0.5)$. Results for bulk Ar are from [11]. The maximum value on $y$ axis is 0.65 for all experimental data; the data for bulk are in arbitrary units. 
size-dependent effects. To keep calculations tractable we consider only those structures which have complete shells. $\left|\mathrm{FT}\left[k^{2} \chi(k)\right]\right|$ is calculated for each unique absorbing site in the cluster, and the results are weighted by the number of atoms in each such site before being added to obtain the mean $\left|\mathrm{FT}\left[k^{2} \chi(k)\right]\right|$. The results are displayed in Figs. 3 (fcc) and 4 (icosahedral). Owing to the crystalline nature of the fcc structure, it suffices to perform calculations for clusters having 13 and 55 atoms and for an fcc solid. The fivefold icosahedral symmetry does not permit a crystalline structure and calculations are performed only for clusters having 13, 55, 147, and 309 atoms. Figure 4 shows that this is sufficient to discern the size-related trends.

The experimentally determined MLRDFs for the larger clusters in Fig. 2 are very similar to that determined for solid $\mathrm{Ar}$ in an earlier work [11] and presented in the same figure. This is an additional indication that these data probe the entire range of interest. Further insight is gained by comparing these functions for different mean cluster sizes. In each case a prominent peak is observed at $r \approx 3.2 \AA$, and it corresponds to the first coordination shell of radius $\approx 3.72 \AA$. Fitting of these data with FEFF theoretical standards shows that the position of this peak is almost independent of the cluster size, which is consistent with the similar value of the $\mathrm{Ar}-\mathrm{Ar}$ bond length in an Ar dimer (3.76 $\AA$ [14]) and solid Ar (3.72 [10]). Moreover, the area under this peak depends on the mean value of the first coordination number for the cluster and shows a gradual enhancement with cluster size. This is attributed to the reduced fraction of the less-coordinated surface atoms in large clusters. Another factor that contributes to this observation is the increased disorder of Ar-Ar bonds with decreasing cluster size. For example, in $\operatorname{Ar}_{\langle 30\rangle}$ the mean-square relative displacement amounts to $\sigma^{2}=0.040(2) \AA^{2}$ compared to 0.032(2) $\AA^{2}$ for $\operatorname{Ar}_{\langle 2900\rangle}$, and both values are larger than that in the solid [10].

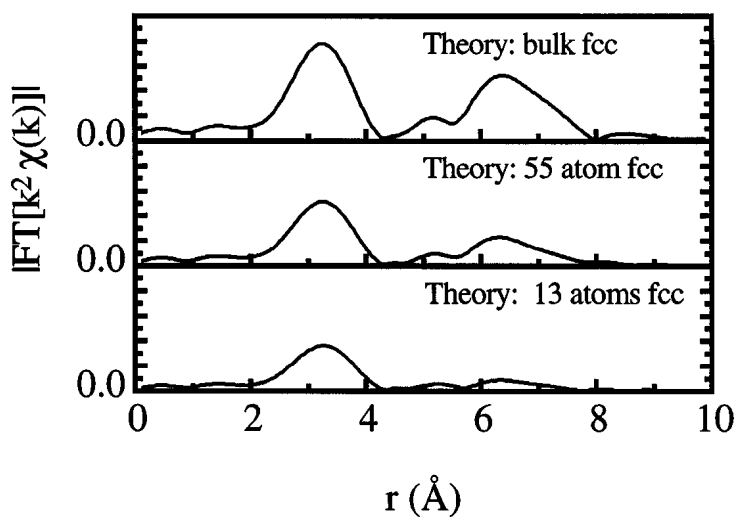

FIG. 3. Theoretically simulated dependence of the mean radial distribution function $\left|\mathrm{FT}\left[k^{2} \chi(k)\right]\right|$ on the cluster size assuming an fcc structure.
To draw conclusions regarding the structure of clusters in the beam, we compare the experimental results with theoretical simulations presented in Figs. 3 and 4. The theoretical results show the peak corresponding to the first coordination shell at low $r$ values. However, our attention is drawn to the feature at $r \approx 6.5 \AA$ in the simulation for the fcc clusters. This feature is ascribed to the scattering of the photoelectron from atoms located in the fourth coordination shell of the absorber. The strength of this feature relative to the first coordination shell peak is contrary to the general expectation that EXAFS contributions from higher shells are strongly damped [1]. However, the reason in this case is well known. The fourth coordination shell in an fcc lattice has twelve atoms, and each of these atoms is collinear with the absorbing atom and one of the twelve atoms in the first coordination shell. This causes strong forward focusing of the photoelectron wave by the atoms in the first coordination shell with the result that contributions from the fourth coordination shell are significantly enhanced. Moreover, every bulk atom in an fcc cluster forms 12 collinear sets of the type described above, which is the maximum possible. This is precisely the origin of the enhancement seen in fourth-shell contributions in Fig. 3. These contributions become even more prominent for large clusters because the fraction of atoms with incomplete fourth coordination shell is diminished. The situation is, however, completely different for the multishell icosahedral clusters. Though the theoretical simulations in Fig. 4 show contributions from coordination shells beyond the first, they are very much subdued compared to the fcc case. This is because in this case the collinearity condition outlined above is satisfied to a much lesser extent, especially along the radial direction. In addition, the tangential bond length in an icosahedral is about 5\% longer than the radial bond length [15] which tends to broaden the peaks at the

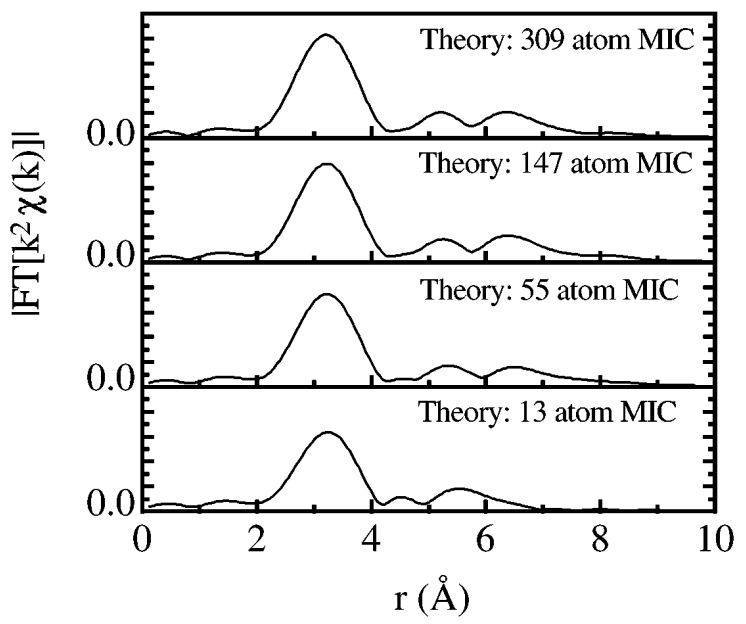

FIG. 4. Theoretically simulated dependence of the mean radial distribution function $\left|\mathrm{FT}\left[k^{2} \chi(k)\right]\right|$ on the cluster size assuming a multishell icosahedral structure (MIC). 
expense of their height, and the effect is more pronounced for contributions from higher cluster shells.

The foregoing discussion allows us to draw conclusions regarding the structure of free Ar clusters. The experimentally determined MLRDFs for these clusters show imprints that are consistent with an fcc structure when the mean cluster size is around 200 or more. These imprints appear in the form of the relative peak heights and the development of feature at $r \approx 6.5 \AA$ in Figs. 2-4. This conclusion is further supported by the gradual evolution of the distribution function and the close resemblance between the results for the largest clusters and those for the solid from [10,11]. For small clusters, it seems that the icosahedral and fcc structures are competing pathways. This is consistent with the fact that the energy differences between the two structures are small [3] and may be subject to strong influences arising from the finite cluster temperature [2] and the vacancies in the cluster shells. However, the transition cluster size of $\langle N\rangle \approx 200$ is much smaller than the theoretical estimates based on considerations of binding energy which range from 1500 to $10^{4}$ [3]. This indicates that energy considerations do not exclusively guide the choice of cluster structure. In addition, a structural transition from icosahedral to fcc at these sizes will require large activation energy and would be probably mediated by a liquid. This has never been observed or simulated and seems unlikely. An alternative hypothesis governing the cluster structure has been advanced by van de Waal and encompasses the plausibility of cluster growth based on the cross-twinning model $[4,5]$. Within this framework it is proposed that rather than the cluster undergoing a glass-crystal transition, a noncrystalline cluster with fivefold symmetry initiates the growth of a nearly perfect fcc crystal by providing favorable sites for stacking faults and remains embedded as the crystal grows, thereby precluding a structural transition. Thus, it is implied that small Ar clusters have predominantly pentagonal structures, a fact consistent with the present results. As the EXAFS contains local structural information, the MLRDFs in Fig. 1 suggest that in small clusters the atoms are embedded mostly in an icosahedral fivefold symmetry. With increasing size, the contribution of these noncrystalline nuclei becomes less important, and the MLRDFs accordingly reflect the dominance of atoms in an fcc environment.

In conclusion, we have presented size-dependent EXAFS data for free $\mathrm{Ar}$ clusters. The data yield size-dependent structural effects with fcc imprints predominating once the mean cluster size in the cluster beam exceeds $\langle N\rangle \approx 200$. This result cannot be reconciled with a picture based solely on energetics. However, it is consistent with the view that the cluster growth mechanism is the guiding factor in structure determination and that particles of fivefold symmetry serve as nuclei for crystalline growth. Consequently, this study provides further impetus to theoretical efforts exploring the link between the cluster structure and growth. Moreover, the potential of EXAFS spectroscopy as a structural tool in cluster physics is clearly displayed. With ongoing advances in cluster generation as well as synchrotron radiation, this technique holds the promise of being as successful in this field as it has been in materials science.

*Electronic address: kakar@vxdesy.desy.de

†Present address: Department of Physics, Uppsala University, Box 530, S-75121 Uppsala, Sweden.

*Visitor: from Laboratorio Nacional de Luz Sincrotron, 13081 Campinas SP, Brazil.

${ }^{\S}$ Present address: Angewandte Physik, Universität Düsseldorf, D-40225 Düsseldorf, Germany.

***Present address: Fachbereich Physik, Universität Osnabrück, Barbarastrasse 7, D-49069 Osnabrück, Germany.

[1] See, e.g., X-Ray Absorption. Principles, Applications, Techniques of EXAFS, SEXAFS and XANES, edited by D.C. Koningsberger and R. Prins (Wiley, New York, 1988).

[2] J. Farges, M.F. de Feraudy, B. Raoult, and G. Torchet, J. Chem. Phys. 78, 5067 (1983); 84, 3491 (1986); Adv. Chem. Phys. 70, 45 (1988).

[3] B. M. Smirnov, Chem. Phys. Lett. 232, 395 (1995); B. W. van de Waal, J. Chem. Phys. 90, 3407 (1989); J. Xie, J.A. Northby, D. L. Freeman, and J. D. Doll, J. Chem. Phys. 91, 612 (1989); B. Raoult, J. Farges, M.F. de Feraudy, and G. Torchet, Philos. Mag. B 60, 881 (1989); J. W. Lee and G. D. Stein, J. Phys. Chem. 91, 2450 (1987).

[4] B. W. van de Waal, Z. Phys. D 20, 349 (1991); J. Chem. Phys. 98, 4909 (1993).

[5] B. W. van de Waal, Phys. Rev. Lett. 76, 1083 (1996); J. Cryst. Growth 158, 153 (1996).

[6] E. Rühl et al., J. Chem. Phys. 98, 6820 (1993); E. Rühl et al., J. Chem. Phys. 98, 2653 (1993); E. Ruḧl et al., Jpn. J. Appl. Phys. 32, Suppl. 32-2, 791 (1993).

[7] F. Federmann, O. Björneholm, A. Beutler, and T. Möller, Phys. Rev. Lett. 73, 1549 (1994); O. Björneholm et al., Surf. Rev. Lett. 3, 299 (1996).

[8] R. Karnbach et al., Rev. Sci. Instrum. 64, 2838 (1993).

[9] E. Rühl et al., J. Chem. Phys. 95, 6544 (1991).

[10] W. Malzfeldt, W. Niemann, P. Rabe, and R. Haensel, Springer Proc. Phys. 2, 445 (1984); W. Malzfeldt, Ph.D. thesis, University of Kiel, 1985.

[11] W. Niemann et al., Phys. Rev. B 35, 1099 (1987).

[12] E. A. Stern et al., Physica (Amsterdam) 208-209B, 117 (1995).

[13] J. J. Rehr, R. C. Albers, and S. I. Zabinsky, Phys. Rev. Lett. 69, 3397 (1992).

[14] P. R. Herman, P.E. LaRocque, and B.P. Stoicheff, J. Chem. Phys. 89, 4535 (1988).

[15] A. L. Mackay, Acta Crystallogr. 15, 916 (1962). 